\title{
Photocrystallographic studies of novel nickel (II) nitro complex supplemented by IR spectroscopy and multi-temperature XRD experiments
}

\author{
P.Borowski ${ }^{1}$, S.E.Kutniewska ${ }^{1}$, R.Kamiński ${ }^{1}$, A. Krówczyński ${ }^{1}$, D. Schaniel ${ }^{2}$, K.N.Jarzembska ${ }^{1}$ \\ ${ }^{1}$ Department of Chemistry, University of Warsaw, Żwirki i Wigury 101, 02-089 Warsaw, Poland \\ ${ }^{2}$ Université de Lorraine \& CNRS, Vandoeuvre-les-Nancy, France \\ pz.borowski2@student.uw.edu.pl
}

The importance of transition-metal switchable compounds of real-life applications is rapidly increasing, thus investigations of nickel (II) organic complexes in which metal centre is coordinated by molecular fragments that can exist in multiple isomeric forms (e.g. $\mathrm{NO}_{2}, \mathrm{SO}_{2}, \mathrm{~N}_{2}$ ) cannot be overestimated. Hence, the current study was devoted to obtain novel promising photoswitchable materials (Fig.1a) characterized by desired reversibility, high conversion percentage and stability.

The designed model compound is shown in Figure 1. As can be seen, the nickel(II) centre is coordinated by the ambidentate nitro ligand and the (N,N,O)-donor moiety. For the purpose of thorough analysis of its photoswitchable properties and the isomerisation reaction features, IR spectroscopy, multi-temperature and photocrystallographic XRD experiments were conducted and supported by computational investigations. Optimal photoisomerisation conditions were determined on the basis of multi-temperature XRD and spectroscopic experiments.<smiles>CC1=CN2CO[N+]([N+](=O)[O-])(OC1c1ccccc1)[n+]1ccccc1C2</smiles>

a)

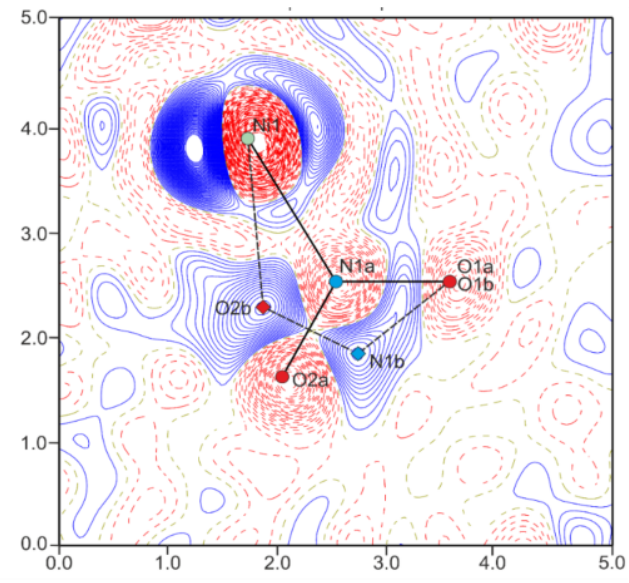

b)

Figure 1. (a) The studied compound. (b) The photodifference map showing the coexsistence of both nitro and exo-nitrito linkage isomers.

The studied complex crystallises in the P-1 space group with one molecule in the asymmetric unit. Upon visible light irradiation the nitro isomer transforms to the endo-nitrito form reaching about $25 \%$ conversion in the form of a single crystal sample and $100 \%$ as a powder according to the IR spectroscopy results. The generated metastable state species exist up to $200 \mathrm{~K}$. Intermolecular contacts, linkage isomers' relative stability, reaction cavity volumes and crystal packing were thoroughly investigated to understand the nitronitrito linkage isomerisation mechanism.

[1] Kamiński, R, Jarzembska, K. N., Kutyła, S. E. \& Kamiński, M. (2016). J.Appl.Cryst.. 49, 1383.

[2] Hatcher, L. E., Skelton, J. M., Warren, M. R. \& Raithby, P. R. (2019). Acc. Chem. Res. 52, 4, 1079.

\section{Keywords: photocrystallography; nickel (II) nitro complexes; photoswitchable compounds}

The authors thank the PRELUDIUM grant (2017/25/N/ST4/02440) of the National Science Centre in Poland and the Inter-Faculty of Individual Studies in Mathematics and Natural Sciences, University of Warsaw, for financial support. The Wroctaw Centre for Networking and Supercomputing (grant No. 285) is gratefully acknowledged for providing computational facilities. The in-house Xray diffraction experiments were carried out at the Department of Physics, University of Warsaw, on Rigaku Oxford Diffraction SuperNova diffractometer, which was co-financed by the European Union within the European Regional Development Fund (POIG.02.01.00-14.122/09) 